Recibido: 30 de julio de 2018

Aceptado: 26 de noviembre de 2018

Publicado en línea:

Doi: 10.32685/2590-7468/invapnu-

clear.2.2018.56
Palabras clave: Método del tripe monitor, análisis por activación neutrónica (AAN), flujo de neutrones, reactor nuclear de investigación, combustible Triga.

Keywords: Triple monitor method, neutron activation analysis (NAA), neutron flux, nuclear research reactor, Triga core.

\section{Implementación del método del triple monitor para la caracterización del flujo neutrónico del reactor nuclear de investigación IAN-R1}

\section{Implementation of the triple monitor method for neutron flux characterization at IAN-RI nuclear research reactor}

Oscar Alberto Sierra ${ }^{1}$, Karel Giovanny Núñez ${ }^{1}$, Fabio Nelson Acero ${ }^{1}$, David Leonardo Alonso ${ }^{1}$

Citación: O. A. Sierra, K. G. Núñez, F. N. Acero y D. L. Alonso, "Implementación del método del triple monitor para la caracterización del flujo neutrónico del reactor nuclear de investigación IAN-R1", Revista de Investigaciones y Aplicaciones Nucleares n. ${ }^{\circ}$ 2, pp. 35-42, 2018.

\section{RESUMEN}

El presente trabajo describe el procedimiento llevado a cabo para la determinación preliminar de parámetros característicos del flujo de neutrones del reactor nuclear de investigación IAN-R1 (RNI IAN-R1) por medio el método del triple monitor no cubierto en la posición de irradiación de periferia del núcleo. Mediante este método se estimó el valor de flujo térmico $\left(\phi_{\text {th }}\right)$, el factor de simetría de flujo de neutrones epitérmicos $(\alpha)$ y la razón entre flujo de neutrones térmicos con respecto al flujo de neutrones epitérmicos $(f)$. Dichos parámetros fueron obtenidos mediante la irradiación de monitores de zirconio $(\mathrm{Zr})$ y de una aleación de oro con aluminio ( $\mathrm{Au}-\mathrm{Al}$ 0,1 \% $\mathrm{Au}$ ), que fueron irradiados en las posiciones de irradiación G3 y G4 del RNI IAN-R1. Como resultado se encontraron los siguientes valores en los parámetros estimados a una potencia de operación de $30 \mathrm{~kW}, \phi_{\mathrm{th}}=2,1 \times 10^{11} \mathrm{~cm}^{-2} \cdot \mathrm{s}^{-1}$ (coeficiente de variación $C V 4 \%), \alpha=0,02$ ( $C V 83 \%)$, y $f=67$ (CV $8 \%$ ). La alta variación de $\alpha$ se explica porque el método usa únicamente tres reacciones de captura para describir el comportamiento de los neutrones en la región epitérmica del espectro. Esta variación se puede mejorar mediante la aplicación de métodos multimonitor para la caracterización de flujo neutrónico.

\section{A BST RACT}

The present work describes the procedure carried out for the preliminary determination of neutron flux parameters for the nuclear research reactor IAN-R1 (RNI IAN-R1) through the not covered triple monitor method in the nucleus peripheral irradiation position. Using this method, the thermal flux value $\left(\phi_{\text {th }}\right)$, the epithermal neutron flux symmetry factor $(\alpha)$ and the ratio between thermal neutron flux with respect to the epithermal neutron flux $(f)$ were estimated. Those parameters were obtained by irradiating zirconium ( $\mathrm{Zr}$ ) monitors and a gold-aluminum alloy monitors ( $\mathrm{Au}-\mathrm{Al} 0.1 \% \mathrm{Au}$ ), which were irradiated at the G3 and G4 irradiation positions of the RNI IAN-R1. The following values were found for the parameters estimated at an operating power of $30 \mathrm{~kW}, \phi_{\text {th }}=2,1 \times 10^{11} \mathrm{~cm}^{-2} \cdot \mathrm{s}^{-1}$ (variance $C V 4 \%$ ), $\alpha=0,02$ $(C V 83 \%)$, and $f=67(C V 8 \%)$. The high variance in $\alpha$ could be explained if we consider that the method only uses 3 capture reactions to describe the epithermal neutron spectrum. The variance could be improved by application of multimonitor methods for neutron flux characterization.

${ }^{1}$ Servicio Geológico Colombiano, Bogotá, Dirección de Asuntos Nucleares

Email de correspondencia: osierra@sgc.gov.co 


\section{INTRODUCCIÓN}

$\boldsymbol{E}$ n Colombia se cuenta con un único reactor nuclear de investigación, denominado IAN-R1, que fue comisionado en 1965, mediante el programa Átomos para la Paz del gobierno de los Estados Unidos [1]. En la actualidad el reactor está adscrito al Servicio Geológico Colombiano, institución que desde el año 2005 ha auspiciado el aprovechamiento de esta instalación para el desarrollo de aplicaciones en el ámbito de la geología, incluyendo el análisis por activación neutrónica $(\mathrm{AAN})$, que permite la determinación química multielemental de materiales, el análisis de huellas de fisión (HF) para la datación de eventos geológicos y el conteo de neutrones retardados (NR) para la determinación cuantitativa de uranio (U) y torio (Th) en materiales geológicos.

En lo que respecta a sus características técnicas, el IAN-R1 se clasifica como un reactor tipo piscina, moderado por el componente hidrogenado de su combustible, reflejado por grafito y enfriado por convección natural de agua ligera de baja conductividad. El núcleo del reactor es tipo Triga, con un enriquecimiento en ${ }^{235} \mathrm{U}$ cercano al $20 \%$, con una potencia máxima de operación de $30 \mathrm{~kW}$, lo que permite obtener un flujo de neutrones del orden de $10^{11} \mathrm{~cm}^{-2} \cdot \mathrm{s}^{-1}[2]$.

El flujo de neutrones en las diferentes posiciones de irradiación de un reactor nuclear de investigación se encuentra caracterizado por dos parámetros principales: el primero, denominado $f$, se define como la relación entre el flujo neutrónico térmico a epitérmico $\left(\phi_{\mathrm{th}} / \phi_{\mathrm{epi}}\right)$; el segundo se conoce como parámetro $\alpha$, y mide la desviación del comportamiento $1 / E$ (donde $E$ es la energía del neutrón) en los neutrones en la región epitérmica del espectro de neutrones [3], [4], lo cual supone que el flujo de los neutrones en esta región del espectro disminuye con el aumento de la energía del neutrón (figura 1). La determinación de estos dos parámetros involucra la calibración del canal de irradiación.

En la literatura se describen tres metodologías básicas para realizar la calibración experimental de los canales de irradiación: 1) el método de relación de cadmio multimonitor, 2) el método de tres monitores no cubiertos (típicamente ${ }^{197} \mathrm{Au},{ }^{94,96} \mathrm{Zr}$ ), y 3) el método de multimonitor cubierto con Cd [3,5-7]. Aunque otras alternativas también han sido exploradas, como el uso de un sistema multimonitor de (Au, Cr, Mo) [8], cada una de estas metodologías implica la irradiación de sistemas de múltiples monitores y estrictas rutinas de medición, con el fin de obtener una menor incertidumbre en la estadística de conteo de los fotones emitidos por los radionúclidos formados durante el proceso de captura de neutrones.

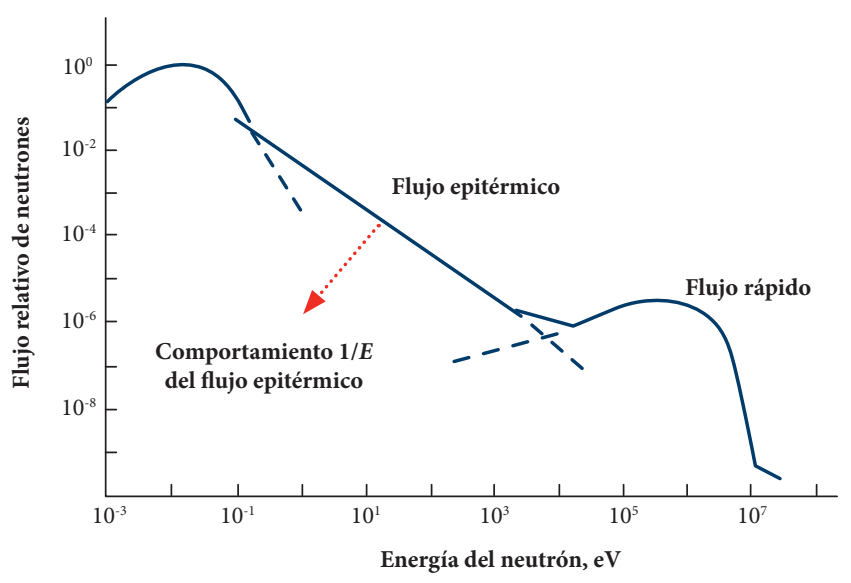

Figura 1. Esquema del espectro de flujo neutrónico en un reactor nuclear [4].

La importancia de la calibración de los canales de irradiación reside en que sirve como base para el desarrollo de métodos avanzados de calibración para el análisis por activación neutrónica, como $\mathrm{K}_{0, \mathrm{Au}}$ [9]. Por otra parte, el conocimiento de estos parámetros permite establecer las posibles aplicaciones que se pueden desarrollar en determinado canal de irradiación (p. ej., análisis por activación neutrónica instrumental [AANI], análisis por activación neutrónica con neutrones epitérmicos [AANE], huellas de fisión $[\mathrm{HF}]$ y conteo de neutrones retardados [NR], entre otros) [10].

En el caso del reactor colombiano IAN-R1, se han realizado experimentos para determinar el flujo térmico y su distribución axial mediante el uso de una sonda autoenergizada de vanadio (SPND) en las posiciones internas de irradiación del núcleo, en los elementos reflectores de grafito y en la posición de irradiación en periferia [11]. El flujo neutrónico máximo determinado en estos trabajos fue hallado en los canales internos, con un valor cercano a 7,8 $\times 10^{11}$ $\mathrm{cm}^{-2} \cdot \mathrm{s}^{-1}$. En otro trabajo se realizó la estimación del parámetro $f$ y del flujo térmico del reactor para la posición de irradiación en periferia del núcleo, usando como primera aproximación un valor de $\alpha=0$; en esa oportunidad se encontró un valor promedio de flujo térmico de $1,5 \times 10^{11} \mathrm{~cm}^{-2}$ . $\mathrm{s}^{-1} \mathrm{y}$ un valor promedio de $f$ de 36 , con una potencia de operación de $20 \mathrm{~kW}$ [12]. 
Finalmente, en el presente trabajo se realizó la determinación preliminar de los parámetros $f, \alpha$ y $\phi_{\text {th }}$ en la posición de irradiación de periferia del núcleo mediante el método del triple monitor descubierto, en el cual se utilizan tres reacciones de captura de neutrones diferentes para la calibración del canal, descritas en (1) a (3) [3].

${ }^{94} \mathrm{Zr}(\mathrm{n}, \gamma){ }^{95} \mathrm{Zr} ;{ }^{95} \mathrm{Zr} \rightarrow \beta^{-}+{ }^{95} \mathrm{Nb}+\gamma_{\text {característica }}$
${ }^{96} \mathrm{Zr}(\mathrm{n}, \gamma){ }^{97} \mathrm{Zr} ;{ }^{97} \mathrm{Zr} \rightarrow \beta^{-}+{ }^{97} \mathrm{Nb}+\gamma_{\text {característica }}$
${ }^{197} \mathrm{Au}(\mathrm{n}, \gamma){ }^{198} \mathrm{Au} ;{ }^{198} \mathrm{Au} \rightarrow \beta^{-}+{ }^{198} \mathrm{Hg}+\gamma_{\text {característica }}$

De esta manera, de la medición de la radiactividad inducida en monitores de Zr y Al-Au por su exposición a un campo de neutrones, es posible la estimación de los parámetros de calibración del canal de irradiación estudiado.

\section{DESCRIPCIÓN DEL MÉTODO}

\subsection{Método empleado}

El método usado en la estimación de los parámetros de calibración de flujo neutrónico corresponde al triple monitor descubierto, en el cual se hace uso de tres reacciones de captura neutrónica con diferentes secciones eficaces o probabilidades de ocurrencia en la región de energía correspondiente a neutrones térmicos y epitérmicos, considerando que en estos últimos existe una dependencia funcional con la energía del neutrón, indicada en (4) $[13,14]$.

$$
\phi_{\text {epi }}(E)=\phi_{\text {epi }} \frac{1 \mathrm{eV}}{E^{1+\alpha}}
$$

Usualmente, el sistema de monitores escogido en este método corresponde a la irradiación de láminas circulares de pequeño espesor de los metales zirconio $(\mathrm{Zr})$, y de una aleación de bajo contenido de oro con aluminio ( $\mathrm{Au}-\mathrm{Al}$ ). Lo anterior se sustenta en la baja incertidumbre que se tiene sobre las secciones eficaces, la posibilidad de contar con materiales de pureza conocida, la posibilidad que ofrecen los isótopos estables del Zr y el Au para capturar los neutrones con energía en la región térmica y epitérmica del espectro, y en que los radionúclidos formados decaen mediante esquemas sencillos que no involucran emisión de fotones en cascada.
De acuerdo con lo planteado por De Corte y colaboradores [13], usando este set de monitores, y considerando que el flujo de neutrones en la región epitérmica presenta la dependencia con la energía del neutrón indicada en (4), el parámetro $\alpha$ puede ser calculado de acuerdo con lo indicado en (5).

$(a-b) Q_{0,1}(\alpha) \frac{G_{\mathrm{epi}, 1}}{G_{\mathrm{th}, 1}}-a Q_{0,2}(\alpha) \frac{G_{\mathrm{epi}, 2}}{G_{\mathrm{th}, 2}}+b Q_{0,3}(\alpha) \frac{G_{\mathrm{epi}, 3}}{G_{\mathrm{th}, 3}}=0$

Donde

$a=\left(1-\frac{A_{\mathrm{sp}, 2} K_{0, \mathrm{Au}, 1} \mathcal{E}_{\mathrm{p}, 1}}{A_{\mathrm{sp}, 1} K_{0, \mathrm{Au}, 2} \mathcal{E}_{\mathrm{p}, 2}}\right)^{-1} ; b=\left(1-\frac{A_{\mathrm{sp}, 3} K_{0, \mathrm{Au}, 1} \varepsilon_{\mathrm{p}, 1}}{A_{\mathrm{sp}, 1} K_{0, \mathrm{Au}, 3} \varepsilon_{\mathrm{p}, 3}}\right)^{-1}$

$Q_{0, i}(\alpha)=\left(Q_{0, i}-0,429\right)\left(\bar{E}_{r, i}\right)^{-\alpha}+\frac{0,429}{(2 \alpha+1)(0,55)^{\alpha}}$

En estas ecuaciones, los términos $G_{\mathrm{epi}, i}$ y $G_{\mathrm{th}, i}$ corresponden a los factores de autoatenuación de los neutrones epitérmicos y térmicos, respectivamente; $Q_{0,1}(\alpha)$ es el cociente entre las secciones eficaces de captura de los neutrones epitérmicos y térmicos; $A_{s p, i}$ corresponde a la actividad específica inducida por las reacciones de captura $\left(A_{s p, i}=N_{p, i} /\left(t_{m}\right.\right.$ $S D C), N_{p, i}$ cuentas del fotopico, $t_{m}$ tiempo de medición, $\mathrm{S}$ factor de saturación, D factor de decaimiento y D factor de decaimiento durante el conteo); $K_{0, \mathrm{Au}}$ son factores empíricos propios de cada reacción de captura; $\varepsilon_{p, i}$, las eficiencias absolutas del sistema de detección usado en la determinación de $A_{s p, i} ; \bar{E}_{r, i}$ es la energía efectiva de resonancia, y los subíndices 1, 2 y 3 corresponden a los radionúclidos ${ }^{97} \mathrm{Zr},{ }^{95} \mathrm{Zr}$ y ${ }^{198} \mathrm{Au}$, respectivamente. La tabla 1 muestra los parámetros nucleares de las reacciones de captura usadas en el método.

Tabla 1. Parámetros nucleares de las reacciones de captura usadas en el método [3], [8]

\begin{tabular}{cccccc}
\hline Reacción & $\sigma_{\mathrm{o}}\left(\mathbf{1 0}^{-24} \mathrm{~cm}^{2}\right)$ & $\mathrm{Q}_{\mathrm{o}}$ & $\bar{E}_{\mathrm{r}}(\mathrm{eV})$ & $G_{\mathrm{epi}}$ & $G_{\mathrm{th}}$ \\
\hline$(1)$ & 0,053 & 5,05 & 6260 & 0,983 & 1 \\
$(2)$ & 0,0213 & 248 & 338 & 0,973 & 1 \\
$(3)$ & 98,7 & 15,7 & 5,65 & 1 & 1 \\
\hline
\end{tabular}

Adicionalmente, el método plantea que el parámetro $f$ puede ser determinado desde la medición de las áreas de los fotopicos asociados a los radioisótopos del $\mathrm{Zr}$, mediante la relación mostrada en (8). 


$$
f=\frac{G_{\mathrm{epi},} \frac{K_{0, \mathrm{Au}, 1}}{K_{0, \mathrm{Au}, 2}} Q_{0,1}(\alpha)-G_{\mathrm{epi}, 2} \frac{A_{s p, 1}}{A_{s p, 2}} Q_{0,2}(\alpha)}{G_{\mathrm{th}, 2} \frac{A_{s p, 1}}{A_{s p, 2}}-G_{\mathrm{th}, 1} \frac{K_{0, \mathrm{Au}, 1} \varepsilon_{p, 1}}{K_{0, \mathrm{Au}, 2} \varepsilon_{p, 2}}}
$$

Por último, la determinación del flujo térmico de neutrones se puede realizar aplicando el modelo de la activación neutrónica al proceso de captura del ${ }^{197} \mathrm{Au}$, una vez conocidos los parámetros de calibración, según lo mostrado en (9).

$$
\phi_{\mathrm{th}}=\frac{A_{s p, \mathrm{Au}}}{\sigma_{\mathrm{th}}\left(1+\frac{Q_{0}(\alpha)}{f}\right) N_{0, \mathrm{Au}}}
$$

Donde $\phi_{\text {th }}$ es la sección eficaz de captura térmica, y $N_{0, \mathrm{Au}}$ es el número de núcleos blanco de ${ }^{197} \mathrm{Au}$.

\subsection{Materiales y condiciones de irradiación}

En el presente trabajo se tomaron tres parejas de láminas circulares de Zr (99,8 \% de pureza) y de aleación de Au-Al (IRMM 530) con las características indicadas en la tabla 2. Estas parejas fueron acondicionadas en viales de polietileno de $3,4 \mathrm{~cm}^{3}$ en la manera descrita en la figura 2 .

Tabla 2. Características físicas de los metales usados en la determinación de los parámetros de flujo neutrónico

\begin{tabular}{ccccc}
\hline Metal & Pureza & $\begin{array}{c}\text { Diámetro } \\
(\mathrm{mm})\end{array}$ & Espesor (mm) & Masa (mg) \\
\hline $\mathrm{Zr}$ & $99,8 \%$ & 7,1 & 0,15 & 32,0 \\
\hline $\mathrm{Au}-\mathrm{Al}$ & $0,1 \% \mathrm{Au}$ & 8,0 & 0,10 & 13,5 \\
\hline & & & & \\
\hline
\end{tabular}

Figura 2. Disposición de los monitores en los viales de polietileno.
Los viales acondicionados con las parejas de monitores metálicos fueron contenidos en la gradilla para su irradiación, conforme con lo mostrado en la figura 3. De acuerdo con las dimensiones de la gradilla, la separación entre cada pareja de monitores es del orden de $1,5 \mathrm{~cm}$.

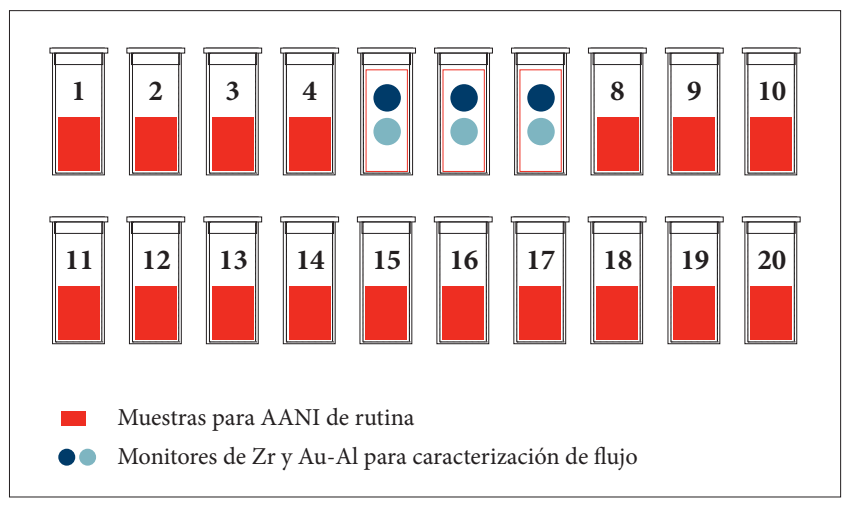

Figura 3. Disposición de los monitores dentro del contenedor de irradiación empleado en las posiciones G3 y G4 de irradiación.

La activación de los materiales se realizó en el reactor nuclear de investigación IAN-R1 (Bogotá, Colombia), en la posición de periferia (G3-G4), operando a una potencia de $30 \mathrm{~kW}$ por un tiempo de irradiación de 4 horas. Para esta posición se han estimado, en trabajos anteriores, flujos neutrónicos del orden de $10^{11} \mathrm{~cm}^{-2} \cdot \mathrm{s}^{-1}$.

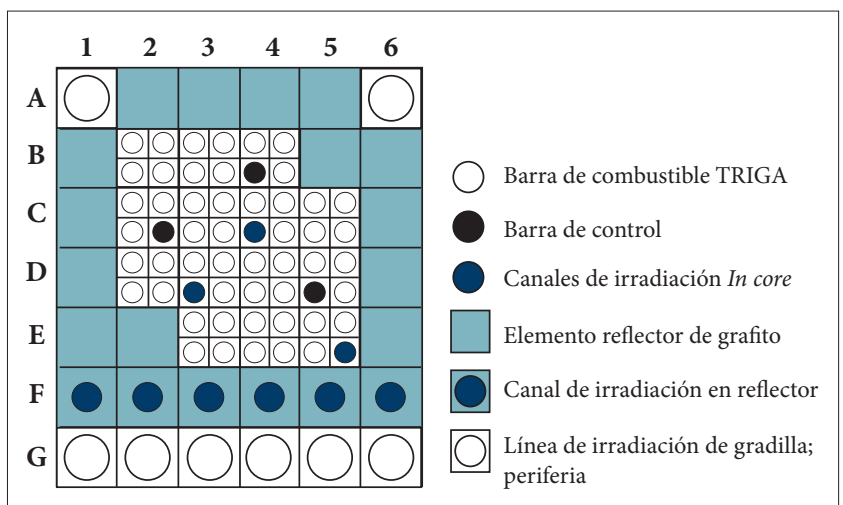

Figura 4. Diagrama del núcleo del reactor IAN-R1.

\subsection{Medición de la actividad inducida por espectrometría gamma}

La medición por espectrometría gamma se realizó en el Laboratorio de Análisis por Activación Neutrónica (LAAN) del SGC, luego de dos días de decaimiento. Para estos efectos, los monitores metálicos irradiados fueron separados individualmente para proceder a su lectura. 
El sistema de detección empleado consta de un espectrómetro gamma con detector de germanio hiperpuro Canberra $^{\circledR}$ GC 7020, y la electrónica asociada al mismo. El software de adquisición y análisis de espectros empleado fue el Genie $2000^{\circledast}$ v.3.3. El espectrómetro fue calibrado en eficiencia a dos distancias fuente del detector, 25 y $13 \mathrm{~cm}$, con fuentes puntuales de actividad certificada. El control del posicionamiento de las muestras se realizó mediante un soporte rígido manual elaborado en acrílico, que permite la ubicación de las muestras sobre el eje central del detector a diferentes distancias.

Para determinar los valores de entrada del modelo de cálculo se usó la rutina de medición especificada en la tabla 3. Los tiempos de medición fueron ajustados para permitir la adquisición de un total de 40000 cuentas en cada uno de los fotopicos de interés.

Tabla 3. Rutina de lectura de los monitores metálicos

\begin{tabular}{cccl}
\hline Lectura & $\mathrm{d}^{1}(\mathrm{~cm})$ & $\begin{array}{c}\text { Radioisótopo } \\
\text { objetivo }{ }^{2}\end{array}$ & \multicolumn{1}{c}{ Uso } \\
\hline L1 & 25 & ${ }^{97} \mathrm{Zr}$ & $\begin{array}{l}\text { Estimación de } b \\
\text { L2 }\end{array}$ \\
& 25 & ${ }^{198} \mathrm{Au}$ & $\begin{array}{l}\text { Estimación de } \\
b \mathrm{y} \phi_{\text {th }}\end{array}$ \\
L3 & 13 & ${ }^{97} \mathrm{Zry}{ }^{95} \mathrm{Zr}$ & $\begin{array}{l}\text { Estimación de } a \\
\alpha \mathrm{y} f\end{array}$ \\
\hline
\end{tabular}

${ }^{1}$ Distancia fuente detector.

${ }^{2}$ Fotones ( $\left.{ }^{97} \mathrm{Zr} 743,4 \mathrm{keV} ;{ }^{95} \mathrm{Zr} 724,2 \mathrm{keV}-756,7 \mathrm{keV} ;{ }^{198} \mathrm{Au} 411,8 \mathrm{keV}\right)$.

\section{RESULTADOS Y DISCUSIÓN}

\subsection{Actividad específica inducida en los monitores}

La figura 5 muestra la comparación de los valores de $A_{s p, i}$ inducida por las reacciones de captura de neutrones sobre las tres parejas de monitores metálicos de $\mathrm{Zr}$ y Al-Au.

La figura 5 muestra una clara tendencia de disminución de la actividad inducida desde la posición 5 a la 7 en las tres reacciones de captura de interés, lo cual pudo haber sido causado por variaciones espaciales del flujo total de neutrones a lo largo del contenedor de irradiación. Esto sugiere que debe considerarse la mejora del contenedor para irradiación de muestras en periferia, ya que este tipo de variación puede incrementar el error en métodos de ensayo como el AANI. Adicionalmente, al revisar los coeficientes de variación de cada serie del radioisótopo formado, se encontró que este no supera el $5 \%$, por lo cual se espera que la variación de flujo de neutrones no sea mayor de $5 \%$.

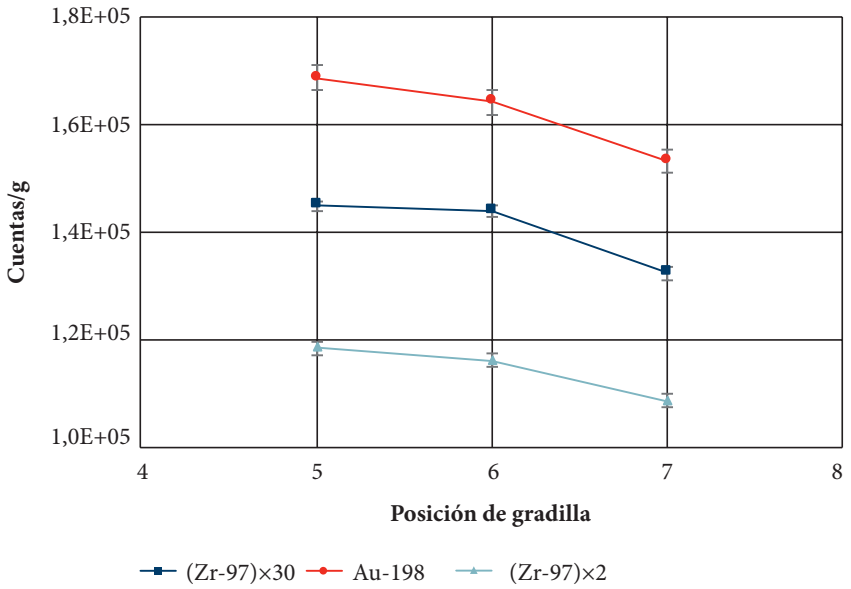

Figura 5. Comparación de las actividades específicas inducidas en los monitores metálicos.

\subsection{Valores determinados para los parámetros a y b}

De acuerdo con lo estipulado en la ecuación 6, los parámetros a y b dependen en gran medida de los valores de $A_{s p, i}$ inducidos en los monitores metálicos. Tal como lo indica la figura 4 , se tienen variaciones significativas en los valores de $A_{s p, i}$ entre las posiciones de irradiación consideradas. Sin embargo, el modelo de cálculo hace uso de los cocientes de las actividades inducidas para la determinación de los parámetros $a \mathrm{y} b$, por lo cual es posible que los cocientes sean similares en cada pareja de monitores. La tabla 4 muestra los valores determinados de $a$ y $b$ en las tres parejas de monitores empleados.

Tabla 4. Parámetros a, b determinados en las posiciones 5 a 7 del contenedor de irradiación

\begin{tabular}{ccc}
\hline Posición & $\boldsymbol{a}$ & $\boldsymbol{b}$ \\
\hline 5 & 1,327 & 1,404 \\
6 & 1,327 & 1,392 \\
7 & 1,336 & 1,400 \\
\hline Promedio & 1,330 & 1,399 \\
Desviación estándar & 0,005 & 0,006 \\
$C V(\%)$ & 0,4 & 0,4
\end{tabular}

La tabla 4 muestra que efectivamente la variación (expresada en $C V=0,4 \%$ ) es baja para la estimación de estos parámetros. 


\subsection{Valores determinados del parámetro de simetría $\alpha$ y de la relación de flujo $f$}

Una vez determinados los valores en $a$ y $b$, es posible determinar el parámetro de simetría en la población de neutrones epitérmicos mediante el uso de la ecuación 5. Para resolver esta ecuación se usó un método de iteración para determinar el mejor valor de $\alpha$ que permite cumplir la condición de igualdad a cero. La tabla 5 muestra los resultados de esta determinación.

Tabla 5. Valores de $\alpha$ y $f$ estimados por el método del triple monitor

\begin{tabular}{ccc}
\hline Posición & $\boldsymbol{\alpha}$ & $\boldsymbol{f}$ \\
\hline 5 & 0,033 & 61 \\
6 & 0,011 & 69 \\
7 & 0,007 & 72 \\
\hline Promedio & 0,02 & 67 \\
Desviación estándar & 0,01 & 6 \\
$C V(\%)$ & 83 & 8 \\
\hline
\end{tabular}

La tabla 5 muestra que los valores estimados de $\alpha$ en cada pareja de monitores resultan ser bastante dispersos ( $\mathrm{CV}$ $=83 \%$ ), a pesar de las bajas dispersiones de los valores de a y b (tabla 4). Esto implica una alta sensibilidad del cálculo de a en este método respecto de variaciones mínimas de los valores de $a$ y $b$ en las relaciones de actividad específica inducida. Este comportamiento ya había sido referenciado por Koster y colaboradores al describir las desventajas del método de $\mathrm{Zr}-\mathrm{Au}[8]$.

Por otra parte, las variaciones en a pueden ser consecuencia de cambios locales del espectro de neutrones a lo largo del contenedor de irradiación.

$\mathrm{Al}$ revisar trabajos realizados en reactores de investigación que han empleado este método de caracterización del flujo de neutrones se evidencia que en geometrías de núcleo tipo TRIGA circulares, con sistema de irradiación rotatorio apagado, las variaciones entre canales vecinos de irradiación pueden ser considerables [15].

Continuando con la aplicación del método, mediante el uso de la ecuación 8 se determinó la relación de flujo térmico a epitérmico $(f)$. El resultado de esta determinación se muestra en la tabla 5. Los resultados muestran que las altas variaciones en $\alpha$ no implican altas variaciones en el valor de $f$ determinado en el método, para el cual se tiene un valor promedio de 67 y una dispersión del 8\%. Para esta posición (G3-G4), el valor obtenido indica que se tiene una buena termalización de los neutrones, lo cual es coherente con posiciones de irradiación en la periferia del núcleo; sin embargo, como confirmación se deben usar otros métodos de caracterización, como $\mathrm{Au} / \mathrm{Au}-\mathrm{Cd}$, para validar el resultado obtenido.

\subsection{Flujo térmico y epitérmico}

Como parte final de la aplicación del método, mediante el uso de la ecuación 9 es posible obtener los valores de $\phi_{\text {th }}$ y $\phi_{\text {epir }}$, usando como entrada los valores determinados de $\alpha, f$ y las actividades específicas inducidas en los monitores de Al-Au.

La tabla 6 muestra los resultados obtenidos correspondientes a la posición de irradiación usada en el presente trabajo.

Tabla 6. Valores de flujo neutrónico determinados por el método

\begin{tabular}{ccc}
\hline Posición & $\phi_{\mathrm{th}}\left(\mathrm{cm}^{-2} \cdot \mathbf{s}^{-1}\right)$ & $\phi_{\mathrm{epi}}\left(\mathrm{cm}^{-2} \cdot \mathbf{s}^{-1}\right)$ \\
\hline 5 & $2,11 \times 10^{11}$ & $3,46 \times 10^{9}$ \\
6 & $2,10 \times 10^{11}$ & $3,04 \times 10^{9}$ \\
7 & $1,98 \times 10^{11}$ & $2,75 \times 10^{9}$ \\
\hline Promedio & $2,1 \times 10^{11}$ & $3,1 \times 10^{9}$ \\
Desviación estándar & $7,2 \times 10^{9}$ & $3,6 \times 10^{8}$ \\
$C V(\%)$ & 4 & 12 \\
\hline
\end{tabular}

El valor de flujo térmico es congruente con lo determinado en trabajos anteriores realizados en el IAN-R1, en los cuales se han reportado valores de $2,3 \times 10^{11} \mathrm{~cm}^{-2} \cdot \mathrm{s}^{-1}$ con una potencia de $30 \mathrm{~kW}$ en posición de irradiación G3-G4 [16], y de $1,53 \mathrm{~cm}^{-2} \cdot \mathrm{s}^{-1}$ con una potencia de $20 \mathrm{~kW}$ [12]. Adicionalmente, en el presente trabajo se reporta por primera vez la magnitud del flujo epitérmico en la posición de irradiación evaluada.

Claramente, la magnitud de $\phi_{\text {th }}$ y de $f$ permite argumentar que esta posición de irradiación es adecuada para el desarrollo de AANI, ya que presenta una buena termalización de los neutrones, con una magnitud de flujo suficiente para su aplicación en la determinación de elementos químicos mayores y a nivel de trazas en muestras geológicas, como lo ha venido usando el SGC [17]; sin embargo, debe trabajarse en la optimización de la geometría del contenedor de irradiación, ya que la forma de gradilla puede causar altas variaciones espaciales de flujo neutrónico a lo largo del contenedor. 


\section{CONCLUSIONES}

Se implementó el método del triple monitor descubierto para caracterizar el flujo neutrónico en el reactor nuclear de investigación IAN-R1. El método permite determinar las características principales del espectro de neutrones $(\alpha, f, y$ $\left.\phi_{\mathrm{th}}\right)$, correspondientes a una posición de irradiación en particular, lo cual es un requisito indispensable para el desarrollo de técnicas avanzadas de calibración en AANI como $\mathrm{K}_{0, \mathrm{Au}}$.

En cuanto a la aplicación del método, se encontró que permite la estimación del parámetro de simetría de la región del espectro de neutrones epitérmicos; sin embargo, la variación de este parámetro resultó ser superior al $80 \%$, lo cual indica una fuerte dependencia del modelo respecto de variaciones pequeñas en las actividades inducidas en los monitores metálicos. Con el objetivo de evaluar estos efectos se debe realizar una evaluación de la incertidumbre asociada a la determinación de $\alpha, f$, y $\phi_{\text {th }}$ en este método.

Por otra parte, la magnitud del flujo térmico de neutrones encontrado $\left(2,1 \times 10^{11} \mathrm{~cm}^{-2} \cdot \mathrm{s}^{-1}\right.$ con un valor de $f$ de 67$)$ sustentaría el uso de la posición de irradiación G3-G3 para el desarrollo de AANI en muestras geológicas, con limitada aplicación en el análisis de elementos traza debido a la baja magnitud de flujo de neutrones. Según esto, se deben caracterizar las demás posiciones de irradiación disponibles, con el fin de encontrar posiciones con un mayor flujo neutrónico y una termalización adecuada.

Finalmente, con el objetivo de validar y complementar la presente implementación, se debe procurar la caracterización del flujo de neutrones mediante técnicas adicionales, como el $\mathrm{Au}-\mathrm{Au} / \mathrm{Cd}$ y el método de relación de cadmio con multimonitor, que permite alcanzar bajas incertidumbres en la determinación del parámetro de simetría de los neutrones epitérmicos [18].

\section{Referencias}

[1] H. Múnera y E. G. Nueva, eds., Tecnología nuclear no medica en Colombia, Bogotá: Tecnicontrol, 2011.

[2] A. International Atomic Energy, Practical Aspects of Operating a Neutron Activation Analysis Laboratory (IAEA-TECDOC). International Atomic Energy Agency, 1990.

[3] F. de Corte y A. Simonits, Vademecum for k0 Users: Addendum to the Kayzero/Solcoi Software Package, publi- cado por DSM Research, Geleen (NL), R94/11492, dic. 1994.[4] R. R. Greenberg, P. Bode y E. A. de Nadai Fernandes, "Neutron Activation Analysis: A Primary Method of Measurement", Spectrochimica Acta - Part B Atomic Spectroscopy, vol. 66, n. 3-4, 2011, pp. 193-241.

[5] D. M. Zangirolami, A. H. Oliveira y A. V. Ferreira, "Thermal and Epithermal Neutron Fluence Rates in the Irradiation Facilities of the TRIGA IPR-R1 Nuclear Reactor", Brazilian Journal of Physics, vol. 40, 2010, pp. 47-51.

[6] M. A. Menezes, A. S. Leal, S. P. Meireles et al., "Neutron Flux Parameters in the TRIGA Mark I IPR-R1 Research Reactor, CDTN/CNEN, for ko-INAA method", en INAC 2013: International Nuclear Atlantic Conference, Brasil, 2013.

[7] K. Embarch, H. Bounouira, M. Bounnakhla et al., "Characterization of Typical Irradiation Channels of Cnesten's TRIGA Mark II Reactor (Rabat, Morocco) Using NAA k0-Method", en 6 African Conference on Research Reactor Utilization and Safety, Nigeria, 2010.

[8] M. J. J. Koster-Ammerlaan, M. A. Bacchi, P. Bode et al., "A New Monitor for Routine Thermal and Epithermal Neutron Fluence Rate Monitoring in k0 INAA", Applied Radiation and Isotopes, vol. 66, n. ${ }^{\circ} 12,2008$, pp. $1964-$ 1969.

[9] R. N. Acharya, A. G. C. Nair, A. V. R. Reddy et al., "Validation of a Neutron Activation Analysis Method Using k0-Standardization", Applied Radiation and Isotopes, vol. 57, n. ${ }^{\circ} 3,2002$, pp. 391-398.

[10]I. A. E. Agency, Applications of Research Reactors. Internat. Atomic Energy Agency, 2014.

[11]J. A. Sarta-Fuentes y L. A. Castiblanco Bohórquez, "Neutron Flux Measurement and Thermal Power Calibration of the IAN-R1 TRIGA Reactor", Proceedings of the 4 . World Triga Users Conference, Francia, 2008.

[12] O. A. Sierra, G. A. Parrado, D. L. Alonso et al., "Estimación de los parámetros de flujo neutrónico $f$ y $\phi$ th a partir de la irradiación de suelos de referencia y monitores de Al-Au", Investigaciones y Aplicaciones Nucleares, vol. 1, n. ${ }^{\circ} 2017,2017$, p. 6.

[13] F. de Corte, L. Moens, K. Sordo-El Hammami et al., "Modification and Generalization of Some Methods to Improve the Accuracy of a-Determination in the $1 /$ E1+a Epithermal Neutron Spectrum", Journal of 
Radioanalytical Chemistry, vol. 52, n. ${ }^{\circ}$ 2, 1 de sep. 1979, pp. 305-317.

[14] J. Ojo y R. Filby, ZAKI: A Windows-Based k o Standardization Code for in-Core INAA, Nucl. Instrum. Meth. Phys. Res., vol. 481, 2002, pp. 502-508.

[15] K. S. Khoo, S. B. Sarmani y I. O. Abugassa, "Determination of Thermal to Epithermal Neutron Flux Ratio $(f)$, Epithermal Neutron Flux Shape Factor $(\alpha)$ and Comparator Factor $(\mathrm{Fc})$ in the TRIGA Mark II Reactor, Malaysia", Journal of Radioanalytical and Nuclear Chemistry, vol. 271, n. ${ }^{\circ} 2,2007$, pp. 419-424.

[16]J.Sandoval, E. López, A. Hernández et al., Thermal Power Calibration and Neutron Flux Measurement of the Nuclear Research Reactor IAN-R1 2015, International Conference on Research Reactors: Safe Management and Effective Utilization. Viena, IAEA, 2015.

[17] G. Parrado, Y. Cañón, M. Peña et al., "Improvement of Analytical Capabilities of Neutron Activation Analysis Laboratory at the Colombian Geological Survey", en AIP Conference Proceedings 1753, 2016.

[18]I. A. Alnour, H. Wagiran, N. Ibrahim et al., "Determination of Neutron Flux Parameters in Puspati TRIGA Mark II Research Reactor, Malaysia", Journal of Radioanalytical and Nuclear Chemistry, vol. 296, n. ${ }^{\circ} 3,2013$, pp. 1231-1237. 